\title{
CEACAM6 induces epithelial-mesenchymal transition and mediates invasion and metastasis in pancreatic cancer
}

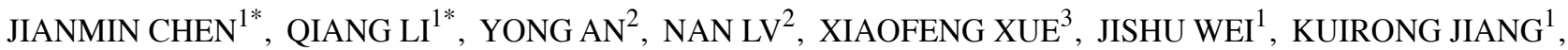 \\ JUNLI WU $^{1}$, WENTAO GAO ${ }^{1}$, ZHUYIN QIAN $^{1}$, CUNCAI DAI $^{1}$, ZEKUAN XU $^{1}$ and YI MIAO ${ }^{1,2}$ \\ ${ }^{1}$ Department of General Surgery, ${ }^{2}$ Laboratory of General Surgery, The First Affiliated Hospital of Nanjing Medical University, \\ Nanjing; ${ }^{3}$ Department of General Surgery, The First Affiliated Hospital of Suzhou University, Suzhou, P.R. China
}

Received April 28, 2013; Accepted June 19, 2013

DOI: 10.3892/ijo.2013.2015

\begin{abstract}
Pancreatic cancer is a disease with an extremely poor prognosis. The acquisition of invasion properties in pancreatic cancer is accompanied by the process of epithelial-mesenchymal transition (EMT). Carcinoembryonic antigen-related cell adhesion molecule 6 (CEACAM6) is emerging as an important determinant of the malignant phenotype in a range of cancers, including pancreatic cancer. Therefore, the aim of this study was to evaluate the potential involvement of CEACAM6 in the invasion and metastasis of pancreatic cancer cells via EMT regulation. The results of our study showed a positive association between CEACAM6 expression and poor prognosis of pancreatic cancer, differentiation and lymph node metastasis. Elevated levels of CEACAM6 in pancreatic cancer cells promoted EMT, migration and invasion in vitro and metastasis in animal models, whereas shRNA-mediated CEACAM6 knockdown had the opposite effect. Furthermore, we demonstrated that miR-29a/b/c specific for CEACAM6 could regulate its expression at the post-transcriptional level. Collectively, our findings identified CEACAM6, which is regulated by $\mathrm{miR}-29 \mathrm{a} / \mathrm{b} / \mathrm{c}$, as an important positive regulator of EMT in pancreatic cancer offering an explanation for how elevated levels of CEACAM6 are likely to contribute to the highly metastatic phenotype of pancreatic cancer.
\end{abstract}

\section{Introduction}

Pancreatic cancer is the most lethal common malignancy, with estimated 43,920 new cases and 37,390 deaths occurring in the United States in 2012 (1). Despite the standardization of surgical techniques and advances in systemic treatments, $<5 \%$

Correspondence to: Dr Yi Miao, Department of General Surgery, The First Affiliated Hospital of Nanjing Medical University, 300 Guangzhou Rd, Nanjing 210029, P.R. China

E-mail: miaoyi@njmu.edu.cn

*Contributed equally

Key words: CEACAM6, pancreatic cancer, epithelial-mesenchymal transition, $\mathrm{miR}-29 \mathrm{a} / \mathrm{b} / \mathrm{c}$ of patients survive 5 years after diagnosis; and this survival rate has remained unchanged for 40 years (2). Furthermore, $<20 \%$ of patients are diagnosed with localized, potentially curable tumors at presentation; while $80-85 \%$ of patients present with an inoperable disease and rapidly succumb to this malignancy (3). In addition, pancreatic cancer responds poorly to most chemotherapeutic agents (3). Hence, there is an urgent need for a better understanding of the molecular mechanisms that contribute to pancreatic cancer development and progression as well as for new potential diagnostic and prognostic tumor markers.

Epithelial-mesenchymal transition (EMT) plays an important role in human physiology and pathophysiology in processes such as organ development, wound healing, organ fibrosis and cancer progression (4-6). This process is accompanied by dramatic changes in cellular morphology, the loss and remodeling of cell-cell and cell-matrix adhesions and the gain of migratory and invasive capabilities (4-7). In pancreatic cancer, induction of EMT leads to acquisition of invasive, metastatic properties as well as chemoresistance (8-10). Therefore, EMT might be an important mechanism involved in pancreatic cancer progression and might contribute to its poor prognosis. All these findings suggest that characterization of EMT effectors is likely to yield new insights into metastasis and novel avenues for treatment of pancreatic cancer.

MicroRNAs (miRNAs) are a class of small non-coding RNAs that post-transcriptionally regulate gene expression by pairing with complementary nucleotide sequences in the 3'-untranslated region (3'-UTR) of target mRNAs (11). Several previous studies have revealed that miRNAs play an important role in EMT and repress target mRNAs through translational downregulation and deadenylation (12-14).

Carcinoembryonic antigen-related cell adhesion molecule 6 (CEACAM6; 19q13.2) is a glycosylphosphatidylinositol (GPI)-linked immunoglobulin superfamily member. There is accumulating evidence that CEACAM6 is overexpressed in several epithelial carcinomas including colon, breast, nonsmall cell lung cancer and intrahepatic cholangiocarcinoma (15-19). In addition, it is involved in many crucial cellular events such as migration, invasion and tumorigenicity $(20,21)$. Recent studies have suggested that CEACAM6 plays important roles in pancreatic cancer development and progression. Indeed, adenocarcinoma gene expression profiling studies 
have shown a 20- to 25-fold higher expression of CEACAM6 compared to normal pancreatic ductal epithelial cells (22). Moreover, deregulated overexpression of CEACAM6 has been shown to inhibit differentiation and anoikis (20). Conversely, knockdown of CEACAM6 has been shown to reverse anoikis resistance and inhibit the metastatic potential in pancreatic cancer mouse xenograft models in vivo by enhancing caspase-3-mediated apoptosis (21). In addition, CEACAM6 gene silencing markedly increased sensitivity to gemcitabinemediated cytotoxicity (23).

Nevertheless, there are no previous studies on the role of CEACAM6 in pancreatic cancer EMT and the mechanisms regulating CEACAM6 expression in tumor progression still remain to be elucidated.

In the present study, we demonstrated that CEACAM6 is an important regulator of pancreatic cancer EMT, migration and invasion in vitro and metastasis in vivo. Furthermore, we showed that CEACAM6 might be a miR-29a/b/c target gene in the pancreatic cancer cell line CFPAC-1.

\section{Materials and methods}

Cell culture. Human pancreatic cancer cell lines CFPAC-1 and PANC-1 were purchased from Shanghai Cell Bank (Shanghai, China) and cultured in Dulbecco's modified Eagle's medium (DMEM) (Invitrogen, Carlsbad, CA, USA) supplemented with $10 \%$ fetal bovine serum (FBS) (Sigma, St. Louis, MO, USA), $2 \mathrm{mM}$ glutamine, $100 \mu \mathrm{g} / \mathrm{ml}$ penicillin and $100 \mu \mathrm{g} / \mathrm{ml}$ streptomycin. All cells were incubated at $37^{\circ} \mathrm{C}$ in a humidified chamber supplemented with $5 \% \mathrm{CO}_{2}$.

Immunohistochemistry. Pancreatic cancer tissue samples were obtained from 99 patients undergoing a pancreatectomy for pancreatic cancer at the First Affiliated Hospital of Nanjing Medical University between 2008 and 2010 and were confirmed by a pathologist. All patients provided informed consent for their participation in the study, which was approved by the Ethics Committee of Nanjing Medical University, China.

For the immunohistochemistry analysis, 4- $\mu \mathrm{m}$ thick paraffin-embedded tissue sections were deparaffinized in xylene, rehydrated in graded alcohol and blocked in methanol containing 3\% hydrogen peroxide. The slides were covered with a blocking solution for $1 \mathrm{~h}$ at room temperature and incubated with mouse anti-human CEACAM6 monoclonal antibody (Abcam, Cambridge, MA, USA) or mouse antihuman E-cadherin monoclonal antibody (Abcam) for $2 \mathrm{~h}$ at $37^{\circ} \mathrm{C}$. After rinsing with phosphate-buffered saline (PBS; $\mathrm{pH}$ 7.4) solution, sections were treated with a goat anti-mouse secondary antibody (Santa Cruz Biotechnology, Santa Cruz, $\mathrm{CA}, \mathrm{USA}$ ) for $1 \mathrm{~h}$ at $37^{\circ} \mathrm{C}$. Next, the slides were incubated with 3,3-diaminobenzidine (DAB) solution for $10 \mathrm{~min}$ and then counterstained with hematoxylin. CEACAM6 and E-cadherin expression were quantified using Image-Pro Plus version 6.0 (Media Cybernetics, Inc., Bethesda, MD, USA).

Generation of stable cell lines. For the generation of stable cell lines in our study, CEACAM6 lentiviral constructs were amplified using PrimeSTAR HS DNA Polymerase (Takara, DR010A, Dalian, China) and ligated into the Lv-CMV-EGFP vector. The shRNAs for human CEACAM6 were designed in our lab and constructed in pLKO.1-puro vectors. Three shRNA plasmids were constructed against different CEACAM6 coding sequence (CDS) regions and a scrambled sequence was made as a negative control. All plasmids were verified by sequencing (Invitrogen). After infection with lentivirus, cells were tested for CEACAM6 gene overexpression or knockdown efficiency. One construct with $\geq 80 \%$ knockdown efficiency was selected and used in further studies. The shRNA sequences used in knockdown studies were as follows: shCEACAM6 (sense: 5'-GCCCCAGAAUCGUAUUGGUTT-3' and antisense: 5'-ACCAAUACGAUUCUGGGGCTT-3') and shCont (sense: 5'-UUCUCCGAACGUGUCACGUTT-3' and antisense: 5'-ACGUGACACGUUCGGAGAATT-3').

Real-time quantitative reverse transcription PCR ( $q R T-P C R)$. For the real-time quantitative RT-PCR analysis, total RNA was extracted using TRIzol reagent (Invitrogen) and cDNA was synthesized using the PrimeScript RT kit (Takara). Real-time quantitative reverse transcription PCR (qRT-PCR) was performed using a FastStart Universal SYBR Green Master (Rox) (Roche, USA) and ABI PRISM 7500 Sequence Detection System (Applied Biosystems, Life Technologies Corp., CA, USA). The relative expression of mRNA was examined as the inverse log of the $\Delta \Delta \mathrm{Ct}$ and normalized to the reference gene, GAPDH. Primers for qPCR were synthesized by Invitrogen (Shanghai, China) and the sequences were as follows: CEACAM6 sense: 5'-AGAAGCTAGCAGAGACCA TGGGACCC-3', antisense: 5'-AAATTCTAGAGGGCTGC TATATCAGAGCC-3'. GAPDH sense: 5'-TCACCCACACTG TGCCCATCTACGA-3', antisense: 5'-CAGCGGAACCGCT CATTGCCAATGG-3'. The other primers are available upon request.

The miR-29a/b/c level was quantified by qRT-PCR using a TaqMan probe (Applied Biosystems, Foster City, CA, USA), with RNU6B small nuclear RNA as an internal reference. Their relative levels were analyzed in triplicate on an ABI PRISM 7900 Sequence Detection System (Applied Biosystems), according to the manufacturer's protocol.

Western blot analysis. For the western blot analysis, cells were lysed using a RIPA buffer with $1 \%$ phenylmethanesulfonyl fluoride (PMSF). Protein concentration was measured using a BCA kit (Keygen, Nanjing, China). Equal amounts of protein $(30 \mu \mathrm{g})$ were resolved with 10\% SDS-PAGE and transferred to polyvinylidene difluoride (PVDF) membranes (Millipore, Bedford, MA, USA). Membranes were probed with primary antibodies for $12 \mathrm{~h}$ at $4^{\circ} \mathrm{C}$ and then incubated with secondary antibodies for $2 \mathrm{~h}$ at room temperature. CEACAM6 (1:250), E-cadherin $(1: 1,000)$, vimentin (1:500) and ZEB1 (1:100) antibodies were from Abcam and the ZEB2 (1:200) antibody was from Santa Cruz Biotechnology. The goat anti-rabbit and goat anti-mouse secondary antibodies were from Beyotime (Nantong, China). GAPDH antibody (1:500) (Beyotime) was used as an internal control. Electrochemiluminescence was performed with a ChemiImager 5500 imaging system (Alpha Innotech Co., San Leandro, CA, USA).

Target prediction and microRNA transfection. Three online programs, TargetScan (http://www.targetscan.org), Microcosm 
Targets (http://www.ebi.ac.uk) and microRNA (http://www. microrna.org) were used for predicting miRNAs that might target CEACAM6. CFPAC-1 cells overexpressing CEACAM6 were used for target miRNA verification. The miRNA mimics were designed and synthesized by Genepharma (Shanghai, China). The miR-29a/b/c mimics and the negative control were as follows: miR-29a sense: 5'-UAGCACCAUCUGAAAUCGG UUA-3' and antisense: 5'-ACCGAUUUCAGAUGGUGCU AUU-3'; miR-29b sense: 5'-UAGCACCAUUUGAAAUCAGU GUU-3' and antisense: 5'-CACUGAUUUCAAAUGGUGCU AUU-3'; miR-29c sense: 5'-UAGCACCAUUUGAAAUCGG UUA-3' and antisense: 5'-ACCGAUUUCAAAUGGUGCU AUU-3'; negative control sense: 5'-UUCUCCGAACGUGUCA CGUTT-3' and antisense: 5'-ACGUGACACGUUCGGAGA ATT-3'.

MicroRNA transfection was performed using Lipofectamine 2000 (Invitrogen). In brief, CPFAC-1 cells were grown in 6-well plates to $50 \%$ confluency before transfection. Total RNA and proteins were extracted at $48 \mathrm{~h}$ post-transfection and used for qRT-PCR and western blot analysis, respectively.

Luciferase reporter assay. For the luciferase reporter assay, reporter plasmids were constructed by ligating 60-bp synthetic oligonucleotides (Invitrogen) containing putative miRNA binding sites from the human CEACAM6 3'-UTR or their mutant versions into XbaI-FseI sites of the pGL3-control vector (Promega, Madison, WI, USA). Cells were plated at $1.5 \times 10^{5}$ cells/well in 24 -well plates $24 \mathrm{~h}$ before transfection. Cells were transfected with $200 \mathrm{ng}$ of luciferase reporter plasmid plus $80 \mathrm{ng}$ of pRL-TK (Promega) in combination with 60 pmol of the microRNA mimics or negative control using Lipofectamine 2000 (Invitrogen). Luciferase activity was measured $48 \mathrm{~h}$ after transfection using the Dual-Luciferase Reporter Assay System (Promega). Firefly luciferase activity was normalized to renilla luciferase activity for each transfected well.

Cell proliferation assay. Cell proliferation was assessed by the MTT assay. Cells were plated at 1,000 cells/well on 96-well plates. Twenty microliters of MTT $(5 \mathrm{mg} / \mathrm{ml})$ was added to each well and plates were incubated for $4 \mathrm{~h}$ at $37^{\circ} \mathrm{C}$, then $200 \mu \mathrm{l}$ of DMSO was added to each well and plates were agitated for $15 \mathrm{~min}$. The optical density (OD) value of each well was determined by measuring the absorbance, respectively, at 492 and $620 \mathrm{~nm}$ (reference). Survival percentage (\%) was calculated relative to the control.

Cell migration and invasion assay. The cell migration assay was performed using $6.5-\mathrm{mm}$ chambers with $8-\mu \mathrm{m}$ pores (Corning, Corning, NY, USA). In brief, cells were seeded in the upper chambers in serum-free DMEM $\left(1 \times 10^{4}\right.$ cells in $200 \mu \mathrm{l}$ ) and $600 \mu \mathrm{l}$ of $10 \%$ FBS-DMEM was added into the lower wells. After $24 \mathrm{~h}$ at $37^{\circ} \mathrm{C}$, cells migrating to the bottom of the membrane were stained with $0.1 \%$ crystal violet in methanol. Images of three random x10 magnification fields were captured from each membrane and the number of migratory cells was counted. For the cell invasion assay, similar inserts coated with Matrigel were used to determine the invasive potential of the cells. All experiments were done in triplicate.
Orthotopic pancreatic tumor xenograft model. Athymic nude mice (BALB/cA-nu (nu/nu)) (4-6-week-old) were purchased from the Nanjing Medical University Animal Center (Nanjing, China). Mice were anesthetized with $2.5 \%$ avertin and the injection site was cleaned with $70 \%$ ethanol. A $1-\mathrm{cm}$ incision was made in the left subcostal region and the pancreas was exposed. A solution of $1 \times 10^{6}$ PANC-1-CEACAM6 or PANC-1-Cont cells in $30 \mu \mathrm{l}$ of PBS was injected into the tail of the pancreas (ten mice per group). The peritoneum and skin were closed with a 4-/T0 surgical suture. Four weeks postinoculation, all surviving mice were sacrificed and evaluated macroscopically for the presence of orthotopic tumors and metastases in the liver. Tumor volumes were determined by the formula: tumor volume $\left(\mathrm{mm}^{3}\right)=$ [length $\left.(\mathrm{mm})\right] \mathrm{x}$ [width $(\mathrm{mm})]^{2} \times 0.52(24)$.

Statistical analysis. All experiments were repeated in triplicate. All values were expressed as mean \pm standard deviation (SD). Statistical significance was determined using the Student's t-test, Kaplan-Meier survival analysis, log-rank test and Spearman correlation using SPSS 17.0 (Chicago, IL, USA). $\mathrm{P}<0.05$ were considered as statistically significant.

\section{Results}

CEACAM6 expression in pancreatic cancer is correlated with clinicopathological characteristics and the EMT marker E-cadherin. In this study, we examined the expression of CEACAM6 in 99 pancreatic tumor tissue samples by immunohistochemistry. Positive CEACAM6 immunohistochemical reaction was localized to the membrane and cytoplasm of tumor cells (Fig. 1A) and was detected in 90.9\% (90/99) of samples. Furthermore, we examined the correlation between CEACAM6 expression and the clinicopathological characteristics of patients. The results of this analysis are summarized in Table I.

In brief, CEACAM6 expression correlated with tumor differentiation and positive lymph node status $(\mathrm{P}<0.05)$; however, no correlation of CEACAM6 expression with patients' age, gender, tumor location, tumor size, perineural invasion, or $\mathrm{T}$ stage was observed $(\mathrm{P}>0.05)$. Furthermore, according to the Kaplan-Meier test, patients with CEACAM6-negative tumors had significantly longer overall survival, compared with those with CEACAM6-positive tumors $(\mathrm{P}<0.05)$ (Fig. 1B).

Additionally, we examined the expression of the EMT marker E-cadherin by immunohistochemistry and correlated it to CEACAM6 expression. A positive immunohistochemical reaction for E-cadherin was observed mainly on membranes of normal glands and cancer cells (Fig. 1C). Pearson correlative analysis indicated significantly negative correlations between CEACAM6 and E-cadherin expression $(\mathrm{P}<0.01)$ (Fig. 1D).

CEACAM6 promotes EMT in pancreatic cancer cells. To determine the potential role of CEACAM6 in regulating EMT in pancreatic cancer, we analyzed the influence of CEACAM6 overexpression and silencing in PANC-1 and CFPAC-1 pancreatic cancer cell lines, respectively.

To analyze the influence of CEACAM6 overexpression, we transfected the CEACAM6 expression vector Lv-CMV-EGFP- 
A

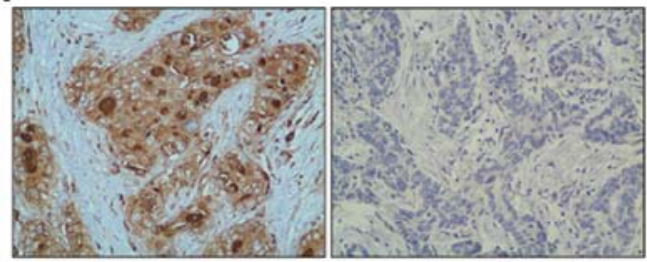

C

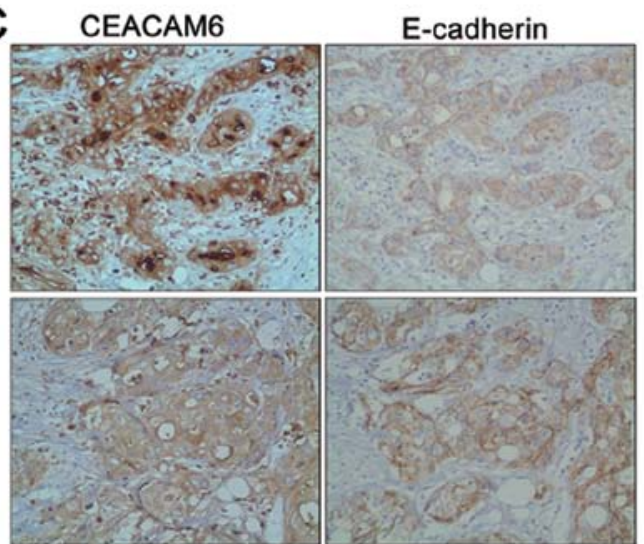

B

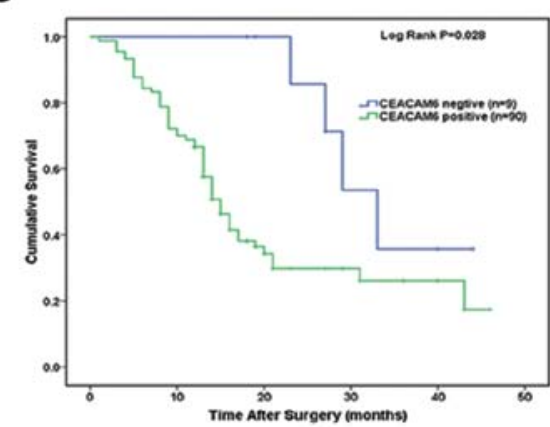

D

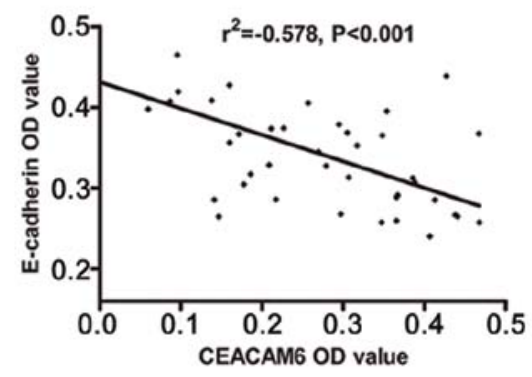

Figure 1. CEACAM6 protein expression in pancreatic cancer specimens and its association with pancreatic cancer pathological features. (A) Pancreatic tumors with positive (left) and negative (right) immunohistochemical reactions for CEACAM6 (magnification, x20). (B) Cumulative survival of pancreatic cancer patients with positive or negative CEACAM6 protein expression. Positive CEACAM6 expression was significantly correlated to shorter survival (P=0.028). Survival analysis was performed using the Kaplan-Meier method. (C) Consecutive immunohistochemical staining of CEACAM6 and E-cadherin protein expression in pancreatic cancer samples (magnification, x20). (D) CEACAM6 expression was negatively correlated with E-cadherin expression in human pancreatic cancer samples.

A

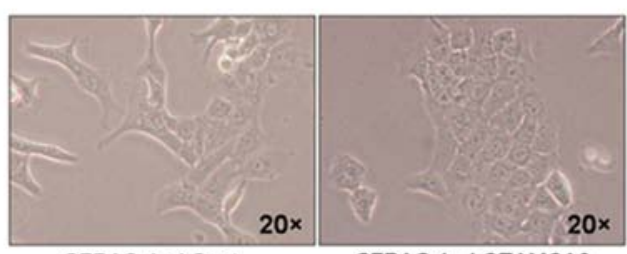

CFPAC-1-shCont

CFPAC-1-shCEAMCA6

B
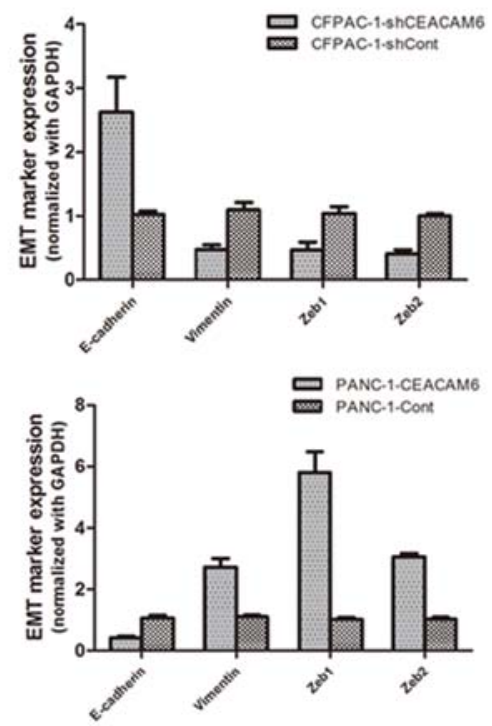

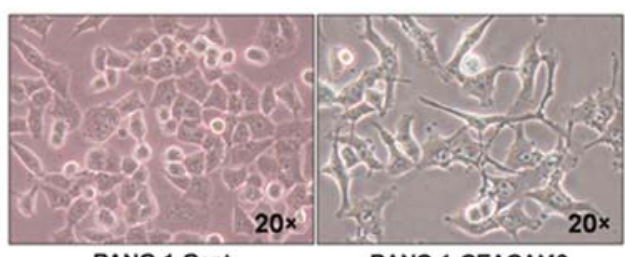

PANC-1-Cont

PANC-1-CEACAM6

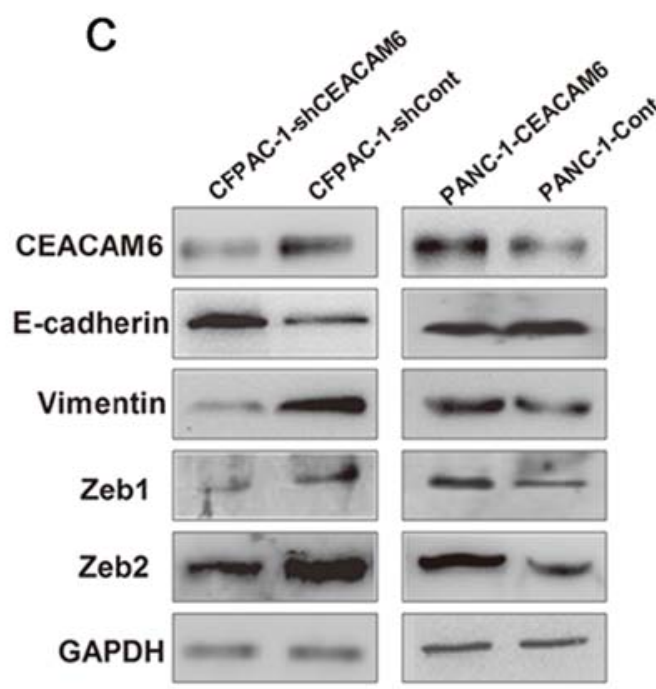

Figure 2. CEACAM6 promotes EMT in pancreatic cancer cells. (A) Phase-contrast photomicrographs. Overexpression of CEACAM6 in PANC-1 cells induced a mesenchymal morphology, whereas knockdown of CEACAM6 in CFPAC-1 cells induced an epithelial morphology (magnification, x20). (B) Real-time qRT-PCR analysis of the mRNA levels of the epithelial marker E-cadherin, the mesenchymal marker vimentin and EMT regulators ZEB1 and ZEB2 in pancreatic cancer cells with CEACAM6 overexpression or knockdown. (C) Western blot analysis of E-cadherin, vimentin, ZEB1 and ZEB2 protein levels in pancreatic cancer cells with CEACAM6 overexpression or knockdown. 
Table I. Association between CEACAM6 imunohistochemical expression and clinicopathological characteristics of pancreatic cancer patients.

\begin{tabular}{|c|c|c|c|}
\hline \multirow[b]{2}{*}{ Characteristic } & \multicolumn{2}{|c|}{ CEACAM6 expression } & \multirow[b]{2}{*}{ P-value } \\
\hline & Positive & Negative & \\
\hline Gender & & & 0.563 \\
\hline Male & 51 & 6 & \\
\hline Female & 39 & 3 & \\
\hline Age (years) & & & 0.703 \\
\hline$\leq 60.8$ & 46 & 4 & \\
\hline$>60.8$ & 44 & 5 & \\
\hline Size $(\mathrm{cm})$ & & & 0.898 \\
\hline$\leq 3.78$ & 52 & 5 & \\
\hline$>3.78$ & 38 & 4 & \\
\hline Differentiation & & & 0.041 \\
\hline Poor & 8 & 0 & \\
\hline Moderate & 75 & 6 & \\
\hline Well & 7 & 3 & \\
\hline Positive lymph nodes & & & 0.019 \\
\hline No & 43 & 8 & \\
\hline Yes & 47 & 1 & \\
\hline Perineural invasion & & & 0.295 \\
\hline No & 25 & 4 & \\
\hline Yes & 65 & 5 & \\
\hline Stage T1/T2/T3 & & & 0.060 \\
\hline $\mathrm{T} 1$ & 19 & 5 & \\
\hline $\mathrm{T} 2$ & 40 & 3 & \\
\hline $\mathrm{T} 3$ & 31 & 1 & \\
\hline Location & & & 0.295 \\
\hline Head & 65 & 5 & \\
\hline Body and limbs & 25 & 4 & \\
\hline
\end{tabular}

CEACAM6 or the control vector Lv-CMV-EGFP into PANC-1 cells, which typically express low levels of CEACAM6. The overexpression of CEACAM6 in PANC-1 cells induced loose cell contact and spindle-shaped morphology reminiscent of EMT, while cells transfected with the control vector maintained the cobblestone-like morphology (Fig. 2A). Next, we observed that elevated expression of CEACAM6 significantly increased the expression of the mesenchymal marker vimentin but decreased the expression of the epithelial marker E-cadherin (Fig. 2B and C, respectively).

Furthermore, in the silencing experiment, we transfected the pLKO.1-puro-shCAECAM6 vector or the control vector pLKO.1-puro-shScramble into CFPAC-1 cells, which typically express high levels of CEACAM6. Knockdown of CEACAM6 in CFPAC-1 cells led to typical transition from mesenchymal to epithelial morphology and a concomitant decrease in vimentin and increase in E-cadherin expression, as evidenced by both qRT-PCR and western blot analysis. Collectively, these findings indicate that altered CEACAM6 expression affects pancreatic cancer cell EMT in vitro.

Furthermore, we examined the levels of the known EMT activators ZEB1 and ZEB2 in relation to CEACAM6 overexpression or knockdown in pancreatic cancer cell lines. ZEB1 and ZEB2 expression was significantly increased in PANC-1 cells overexpressing CEACAM6; whereas in CFPAC-1 cells transfected with CEACAM6, the expression of the silencing vectors $\mathrm{ZEB} 1$ and $\mathrm{ZEB} 2$ was repressed (Fig. 2B and $\mathrm{C}$, respectively). These results suggest a potential role of ZEB1 and ZEB2 in CEACAM6-regulated EMT.

Functional role of CEACAM6 in pancreatic cancer cell proliferation, migration and invasion in vitro. CEACAM6 knockdown and overexpression did not markedly affect the proliferative ability of CFPAC-1 and PANC-1 cell lines (CFPACA-1-shCEACAM6 vs. CFPAC-1-shCont: $0.731 \pm 0.129$ vs. $0.785 \pm 0.119, \mathrm{P}=0.626$; PANC-1-CEACAM6 vs. PANC-1-Cont: $1.293 \pm 0.190$ vs. $1.149 \pm 0.150, \mathrm{P}=0.364$ ) (Fig. 3A and B, respectively). Although the results were not consistent with the impact of altered CEACAM6 expression on proliferation of pancreatic cancer cells in vitro, the data showed that overexpression of CEACAM6 promoted the migration (PANC-1-CEACAM6 vs. PANC-1-Cont: $559.1 \pm 51.9$ vs. $301.6 \pm 36.2, \mathrm{P}<0.01$ ) and invasion (PANC-1CEACAM6 vs. PANC-1-Cont: $437.2 \pm 25.1$ vs. $196.2 \pm 56.2$, $\mathrm{P}<0.01$ ) abilities of PANC-1 cells (Fig. 3D and E, respectively), whereas knockdown of CEACAM6 attenuated cell migration (CFPACA-1-shCEACAM6 vs. CFPAC-1-shCont: 149.7 \pm 30.3 vs. 412.2 $\pm 83.1, \mathrm{P}<0.01)$ and invasion (CFPACA-1-shCEACAM6 vs. CFPAC-1-shCont: $108.2 \pm 27.9$ vs. $354.1 \pm 64.0, \mathrm{P}<0.01)$ in CFPAC-1 cells (Fig. 3C, D and E, respectively).

Overexpression of CEACAM6 enhances metastatic ability of PANC-1 cells in vivo. To assess the significance of CEACAM6 expression in vivo, PANC-1-CEACAM6 cells were orthotopically injected into the pancreas of nude mice, while PANC-1-Cont cells were used as a control. Four weeks after injection, mice were sacrificed and tumor volume and metastatic liver nodules were counted and confirmed histologically (Fig. 4A and B, respectively).

The tumor volume showed no significant difference between the two groups (PANC-1-CEACAM6 vs. PANC-1Cont: $1105.5 \pm 666.7 \mathrm{~mm}^{3}$ vs. $828.5 \pm 439.2 \mathrm{~mm}^{3}, \mathrm{P}=0.286$ ) (Fig. 4C). Nevertheless, a statistically significant difference in the mean metastatic liver nodule number in PANC-1CEACAM6 and PANC-1-Cont groups was observed (5.10 and 0.30, respectively, $\mathrm{P}<0.05$ ) (Fig. 4D).

Modulating effect of miR-29a/b/c on CEACAM6 expression. Using bioinformatic tools (TargetScan, Microcosm Targets and microRNA), we predicted that miR-29a/b/c might be the most potent regulator of the CEACAM6 gene. Therefore, we decided to test our hypothesis in CFPAC- 1 cells using a constructed reporter plasmid carrying the CEACAM6 wildtype and mutant-type 3'-UTR region (Fig. 5A).

As shown in Fig. 5B and C, miR-29a/b/c significantly suppressed luciferase activity when the wild-type 3'-UTR of CEACAM6 was present $(\mathrm{P}<0.05)$. To verify that $\mathrm{miR}-29 \mathrm{a} / \mathrm{b} / \mathrm{c}$ 
A

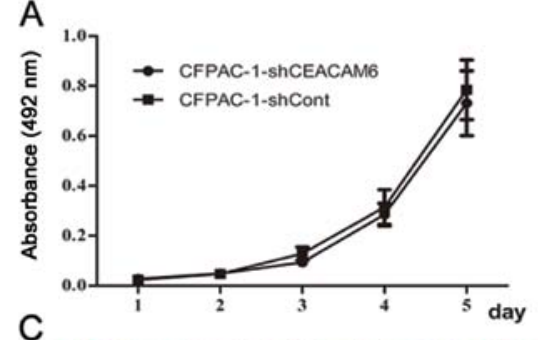

C

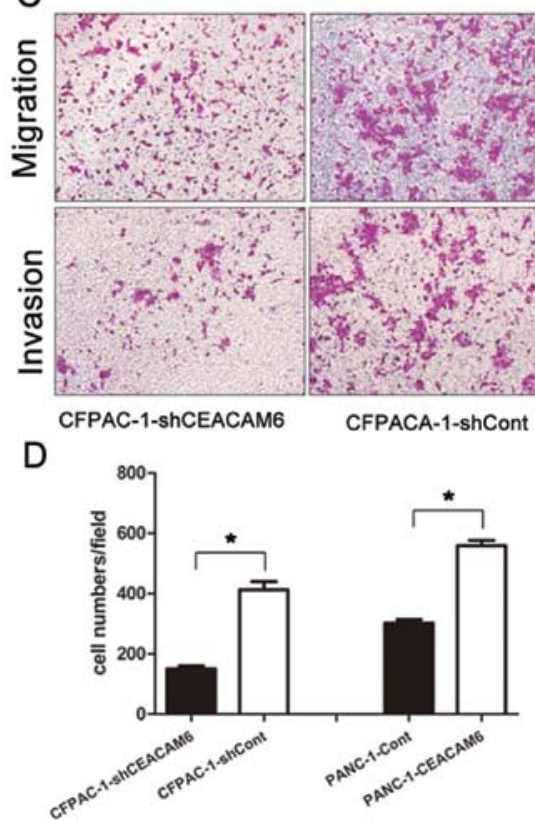

B
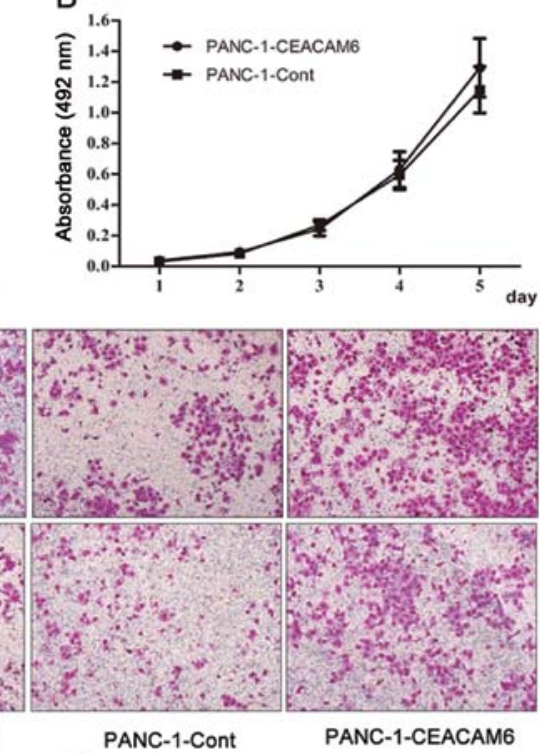

$E$

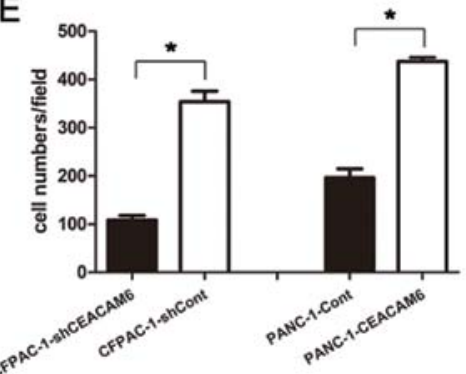

Figure 3. Functional role of CEACAM6 in pancreatic cancer cell proliferation, migration and invasion in vitro. The cell proliferation assay demonstrated that altered CEACAM6 expression did not markedly affect the proliferative ability of transfected CFPAC-1 (A) and PANC-1 cell lines (B). (C) Representative images of migrating or invading pancreatic cancer cells with CEACAM6 overexpression or knockdown (magnification, x10). Average number of migrating (D) or invading cells (E) from three independent experiments. The data are reported as means $\pm \mathrm{SD}$; ${ }^{*} \mathrm{P}<0.05$.
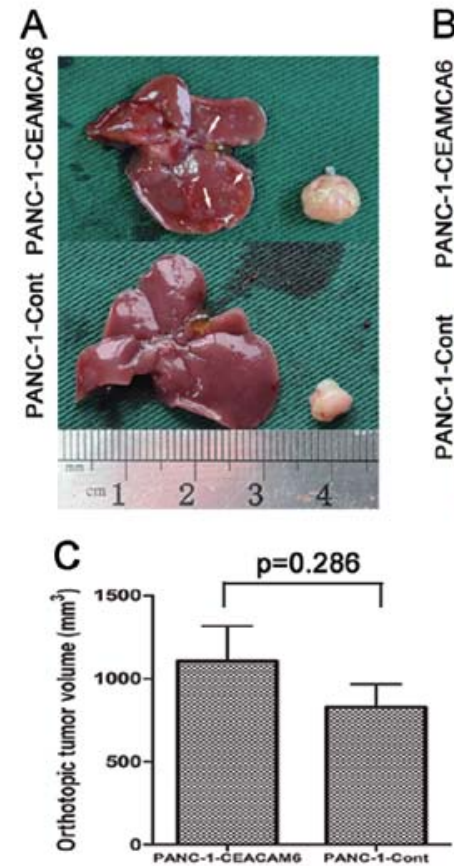

B Pancreas
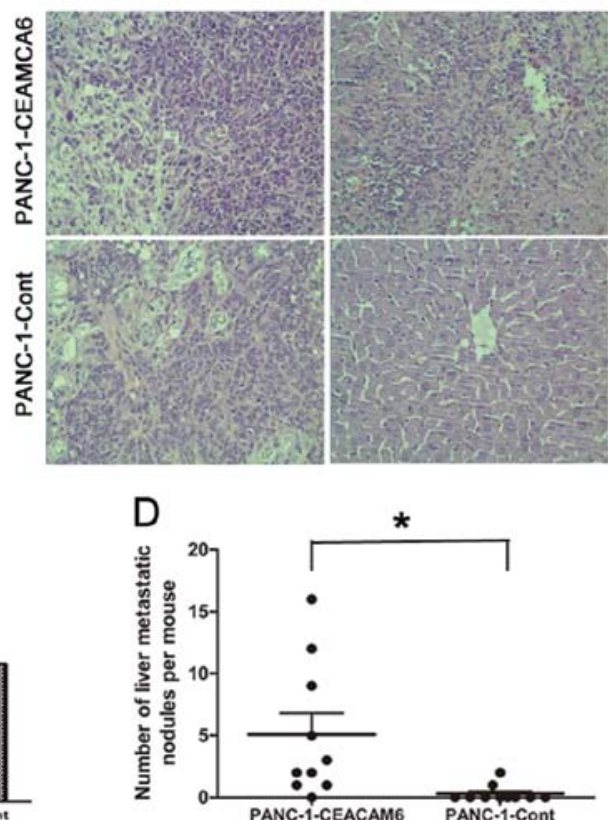

Figure 4. Overexpression of CEACAM6 enhanced the metastatic ability of PANC-1 cells in vivo. (A) Representative images of pancreatic tumors and the corresponding metastatic nodules on the surface of the liver in nude mice at 4 weeks induced by injecting $1 \times 10^{6}$ cells (either PANC-1-Cont or PANC-1-CEACAM6 cells). (B) Examples of hematoxylin and eosin (H\&E) staining of pancreatic tumors and metastatic liver nodules. (C) Comparison of orthotopic tumor volumes after PANC-1-Cont or PANC-1-CEACAM6 cell injection. The data are reported as means $\pm \mathrm{SD}(\mathrm{P}=0.286)$. (D) A scatter plot showing the number of metastatic liver nodules per mouse $(\mathrm{P}<0.05)$. 
A

1104-1111

CEACAM6-3'UTR

5'--AGCGCAUUGAGCCAGUGGUGCUA--3'

miR-29a

| | | | | | | |

miR-29b

3'--AUUGGCUAAAGUCU-.-ACCACGAU--5'

miR-29c

3'--UGUGACUAAAGUUU...-ACCACGAU.-.5'

3'--AUUGGCUAAAGUUU.-.ACCACGAU--5

I $\quad|\quad| X|X X X X|$

CEACAM6-3'UTR-M

5"--AGCGCAUUGAGCCAGUAGCUAGA--3'

B

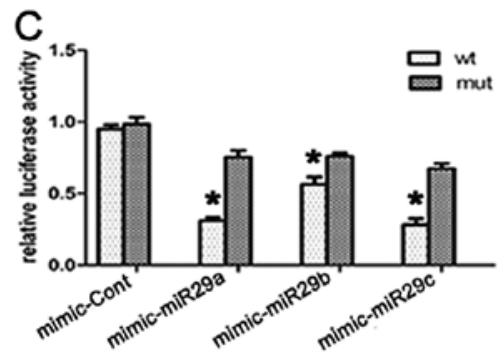

D

E
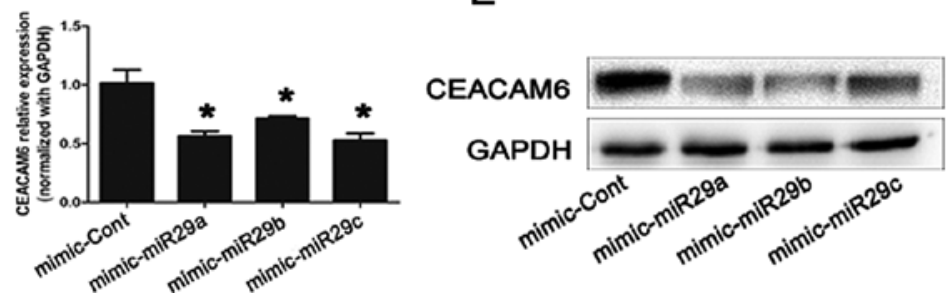

Figure 5. miR-29a/b/c directly targets CEACAM6. (A) The sequence of the CEACAM6 3'-UTR region and its mutant or mismatched sequences. (B) Wild-type (wt) or mutant (mut) CEACAM6-3'-UTR plasmids were transfected into CFPAC-1 cells, with renilla luciferase plasmid (pGL3-cont) as a control. Luciferase activity of plasmids with the wt sequence was suppressed by endogenous miRNAs targeting CEACAM6-3'-UTR ("P<0.05). (C) wt or mut CEACAM6-3'-UTR plasmids were co-transfected with miRNA mimics. The results show that miR-29a/b/c recognizes CEACAM6-3'-UTR and significantly reduces the luciferase activity compared with the mut control ("P<0.05). (D) CEACAM6 mRNA expression in CFPAC-1 cells was analyzed by qRT-PCR at $48 \mathrm{~h}$ post-transfection of miR-29 mimics $(\mathrm{P}<0.05)$. (E) CEACAM6 protein expression in CFPAC-1 cells was analyzed by western blotting at $48 \mathrm{~h}$ post-transfection of miR-29 mimics.

acts as a negative regulator of CEACAM6 translation, we transfected CFPAC-1 cells with miR-29a/b/c mimics and tested the endogenous CEACAM6 mRNA and protein expression levels by qRT-PCR and western blot analysis, respectively. CEACAM6 mRNA levels decreased $48 \mathrm{~h}$ after miR-29a/b/c transfection (Fig. 5D, P<0.05). Additionally, western blot analysis showed that $48 \mathrm{~h}$ after transfection, overexpression of $\mathrm{miR}-29 \mathrm{a} / \mathrm{b} / \mathrm{c}$ resulted in a significant decrease in CEACAM6 protein level (Fig. 5E). These results collectively suggest that $\mathrm{miR}-29 \mathrm{a} / \mathrm{b} / \mathrm{c}$ may, at least in part, be responsible for the regulation of CEACAM6 expression in vitro.

\section{Discussion}

Pancreatic cancer is the tenth most common cancer and the fourth most common cause of cancer mortality worldwide (1). In the past few decades, great efforts have been made to elucidate the molecular mechanisms underlying its tumorigenicity, invasion and metastasis in order to find new potential diagnostic and prognostic markers for early detection as well as to develop new targeted anticancer therapies. Nevertheless, the detailed mechanisms of pancreatic cancer development and progression to metastasis still remain obscure.

Previous studies have shown that CEACAM6 is overexpressed in many carcinomas, including pancreatic cancer $(15,18,19,25)$. It has been suggested that CEACAM6 overex- pression is associated with greater resistance to anoikis and high cellular invasion potential in vitro as well as higher metastatic potential in vivo $(21,26-28)$. The reason why CEACAM6 overexpression is associated with aggressive biological behavior of cancer cells has not been fully clarified.

In the present study, we found that CEACAM6 was highly expressed in most pancreatic cancer tissue samples and this expression was closely associated with poor prognosis in pancreatic cancer patients. In addition, we have for the first time demonstrated that CEACAM6 directly impacts EMT, migration, invasion and metastasis of pancreatic cancer cells. More importantly, our study is the first to show that miR$29 \mathrm{a} / \mathrm{b} / \mathrm{c}$ can regulate CEACAM6 at the post-transcriptional level.

Emerging evidence suggests that EMT is associated with the loss of epithelial and gain of mesenchymal characteristics, resulting in an increased invasive, metastatic and chemo-resistance potential of tumor cells and thus having an important role in cancer progression and prognosis $(29,30)$. In the present study, we found that CEACAM6 is highly expressed in most pancreatic tumor tissues. Clinicopathological analysis revealed that expression of CEACAM6 protein was significantly related to tumor differentiation and lymph node metastasis. Our results are in agreement with those of a previous study by Duxbury et al in which the expression of CEACAM6 correlated with tumor grade and positive lymph node status (25). In 
addition, the observed cell morphology, molecular biomarkers and biological behavior found in our study were consistent with EMT characteristics. Moreover, we demonstrated that elevated CEACAM6 expression could contribute to EMT phenotype acquisition characterized by the typical mesenchymal morphology, through its influence on upregulation of the mesenchymal cell marker vimentin and downregulation of the epithelial cell marker E-cadherin. Conversely, decreased CEACAM6 expression in our study was associated with the reversal of EMT through downregulation of vimentin and upregulation of E-cadherin. Furthermore, these results are consistent with the observed clinical data that showed a significantly negative correlation between CEACAM6 and E-cadherin expression in 40 pancreatic cancer tissues.

ZEB1 and ZEB2, two members of the ZEB family, are important regulators of EMT and are implicated in the tumorigenesis of many human cancers $(12,31)$. We found that ZEB1 and ZEB2 expression was significantly increased in PANC-1 cells overexpressing CEACAM6. On the contrary, ZEB1 and ZEB2 expression was repressed in CFPAC-1 cells in which the CEACAM6 was silenced. Based on these findings, we can speculate on the possible role of CEACAM6 in EMT regulation through its effects on ZEB1 and ZEB2.

The functional study of the role of CEACAM6 in pancreatic cancer cell lines demonstrated that PANC-1 cells, which typically express low levels of CEACAM6 when transfected with CEACAM6 gene, have greater migratory and invasive abilities compared to control-transfected cells. Furthermore, RNA interference-mediated gene suppression of CEACAM6 in the overexpressing pancreatic cancer cell line CFPAC-1 showed marked reduction in migration and invasion capabilities of transfected cells. These findings are consistent with our CEACAM6 immunohistochemistry results as well as in vivo experiments on nude mouse models. In brief, the expression of CEACAM6 in our study was associated with lymph node metastasis in pancreatic cancer patients. Moreover, CEACAM6 overexpression in PANC-1 cells enhanced their ability to form liver metastasis in nude mouse models. Nevertheless, the proliferation ability of pancreatic cancer cells was not affected with either the overexpression or knockdown of CEACAM6 in vitro. This result is further supported by our findings that CEACAM6 overexpression does not influence the orthotopic tumor volume in nude mouse models.

Recent studies in colon cancer, cholangiocarcinoma, hepatocellular carcinoma (HCC) and lung cancer have suggested that miR-29 may have a significant role in tumor biology (32-35). Indeed, Xiong et al have shown that miR-29 expression was reduced in the majority of hepatocellular carcinomas included in their study and its downregulation was significantly associated with poor disease-free survival in HCC patients (36).

In our study, $\mathrm{miR}-29 \mathrm{a} / \mathrm{b} / \mathrm{c}$ overexpression induced a significant downregulation of the CEACAM6 protein and mRNA levels in vitro. In addition, the overexpression of $m i R-29 \mathrm{a} / \mathrm{b} / \mathrm{c}$ was associated with suppression of luciferase-CEACAM63'-UTR activity, indicating that CEACAM6 is a direct target of $\mathrm{miR}-29 \mathrm{a} / \mathrm{b} / \mathrm{c}$.

In conclusion, our results suggest that CEACAM6 plays an important role in the progression and metastasis of human pancreatic cancer by promoting EMT via the ZEB1/
ZEB2 pathway. In addition, we have for the first time shown that $\mathrm{miR}-29 \mathrm{a} / \mathrm{b} / \mathrm{c}$ can regulate CEACAM6 at the post-transcriptional level. Therefore, we conclude that targeting these signaling pathways may be a feasible and effective approach for treatment of pancreatic cancer.

\section{Acknowledgements}

This study was supported by the National Natural Science Foundation of China (NO. NFSC 30972912).

\section{References}

1. Siegel R, Naishadham D and Jemal A: Cancer statistics, 2012. CA Cancer J Clin 62: 10-29, 2012.

2. Vincent A, Herman J, Schulick R, Hruban RH and Goggins M: Pancreatic cancer. Lancet 378: 607-620, 2011.

3. Hidalgo M: Pancreatic cancer. N Engl J Med 362: 1605-1617, 2010.

4. Savagner P: The epithelial-mesenchymal transition (EMT) phenomenon. Ann Oncol 21 (Suppl 7): vii89-92, 2010.

5. Thiery JP, Acloque H, Huang RY and Nieto MA: Epithelialmesenchymal transitions in development and disease. Cell 139: 871-890, 2009.

6. Iwatsuki M, Mimori K, Yokobori T, et al: Epithelial-mesenchymal transition in cancer development and its clinical significance. Cancer Sci 101: 293-299, 2010.

7. Zeisberg $\mathrm{M}$ and Neilson EG: Biomarkers for epithelial-mesenchymal transitions. J Clin Invest 119: 1429-1437, 2009.

8. Rhim AD, Mirek ET, Aiello NM, et al: EMT and dissemination precede pancreatic tumor formation. Cell 148: 349-361, 2012

9. Cano CE, Motoo Y and Iovanna JL: Epithelial-to-mesenchymal transition in pancreatic adenocarcinoma. ScientificWorldJournal 10: 1947-1957, 2010.

10. Krantz SB, Shields MA, Dangi-Garimella S, Munshi HG and Bentrem DJ: Contribution of epithelial-to-mesenchymal transition and cancer stem cells to pancreatic cancer progression. J Surg Res 173: 105-112, 2012.

11. Kim T, Veronese A, Pichiorri F, et al: p53 regulates epithelialmesenchymal transition through microRNAs targeting ZEB1 and ZEB2. J Exp Med 208: 875-883, 2011.

12. Park SM, Gaur AB, Lengyel E and Peter ME: The miR-200 family determines the epithelial phenotype of cancer cells by targeting the E-cadherin repressors ZEB1 and ZEB2. Genes Dev 22: 894-907, 2008

13. Tellez CS, Juri DE, Do K, et al: EMT and stem cell-like properties associated with miR-205 and miR-200 epigenetic silencing are early manifestations during carcinogen-induced transformation of human lung epithelial cells. Cancer Res 71: 3087-3097, 2011.

14. Li Y, Van den Boom TG II, Kong D, Wang Z, Ali S, Philip PA and Sarkar FH: Up-regulation of miR-200 and let-7 by natural agents leads to the reversal of epithelial-to-mesenchymal transition in gemcitabine-resistant pancreatic cancer cells. Cancer Res 69: 6704-6712, 2009.

15. Jantscheff P, Terracciano L, Lowy A, et al: Expression of CEACAM6 in resectable colorectal cancer: a factor of independent prognostic significance. J Clin Oncol 21: 3638-3646, 2003.

16. Maraqa L, Cummings M, Peter MB, et al: Carcinoembryonic antigen cell adhesion molecule 6 predicts breast cancer recurrence following adjuvant tamoxifen. Clin Cancer Res 14: 405-411, 2008.

17. Poola I, Shokrani B, Bhatnagar R, DeWitty RL, Yue Q and Bonney G: Expression of carcinoembryonic antigen cell adhesion molecule 6 oncoprotein in atypical ductal hyperplastic tissues is associated with the development of invasive breast cancer. Clin Cancer Res 12: 4773-4783, 2006.

18. Singer BB, Scheffrahn I, Kammerer R, Suttorp N, Ergun S and Slevogt H: Deregulation of the CEACAM expression pattern causes undifferentiated cell growth in human lung adenocarcinoma cells. PLoS One 5: e8747, 2010.

19. Ieta K, Tanaka F, Utsunomiya T, Kuwano H and Mori M: CEACAM6 gene expression in intrahepatic cholangiocarcinoma. Br J Cancer 95: 532-540, 2006. 
20. Strickland LA, Ross J, Williams S, et al: Preclinical evaluation of carcinoembryonic cell adhesion molecule (CEACAM) 6 as potential therapy target for pancreatic adenocarcinoma. J Pathol 218: 380-390, 2009

21. Duxbury MS, Ito H, Zinner MJ, Ashley SW and Whang EE: CEACAM6 gene silencing impairs anoikis resistance and in vivo metastatic ability of pancreatic adenocarcinoma cells. Oncogene 23: 465-473, 2004.

22. Iacobuzio-Donahue CA, Maitra A, Olsen M, et al: Exploration of global gene expression patterns in pancreatic adenocarcinoma using cDNA microarrays. Am J Pathol 162: 1151-1162, 2003.

23. Duxbury MS, Ito H, Benoit E, Waseem T, Ashley SW and Whang EE: A novel role for carcinoembryonic antigen-related cell adhesion molecule 6 as a determinant of gemcitabine chemoresistance in pancreatic adenocarcinoma cells. Cancer Res 64 3987-3993, 2004.

24. Fu X, Tao L, Li M, Fisher WE and Zhang X: Effective treatment of pancreatic cancer xenografts with a conditionally replicating virus derived from type 2 herpes simplex virus. Clin Cancer Res 12: 3152-3157, 2006.

25. Duxbury MS, Matros E, Clancy T, et al: CEACAM6 is a novel biomarker in pancreatic adenocarcinoma and PanIN lesions. Ann Surg 241: 491-496, 2005.

26. Duxbury MS, Ito H, Benoit E, Zinner MJ, Ashley SW and Whang EE: Overexpression of CEACAM6 promotes insulin-like growth factor I-induced pancreatic adenocarcinoma cellular invasiveness. Oncogene 23: 5834-5842, 2004.

27. Duxbury MS, Ito $\mathrm{H}$, Benoit E, Ashley SW and Whang EE: CEACAM6 is a determinant of pancreatic adenocarcinoma cellular invasiveness. Br J Cancer 91: 1384-1390, 2004.

28. Lewis-Wambi JS, Cunliffe HE, Kim HR, Willis AL and Jordan VC: Overexpression of CEACAM6 promotes migration and invasion of oestrogen-deprived breast cancer cells. Eur J Cancer 44: 1770-1779, 2008.
29. Acloque H, Adams MS, Fishwick K, Bronner-Fraser M and Nieto MA: Epithelial-mesenchymal transitions: the importance of changing cell state in development and disease. J Clin Invest 119: 1438-1449, 2009.

30. Eastham AM, Spencer H, Soncin F, Ritson S, Merry CL, Stern PL and Ward CM: Epithelial-mesenchymal transition events during human embryonic stem cell differentiation. Cancer Res 67: 11254-11262, 2007.

31. Leshem O, Madar S, Kogan-Sakin I, et al: TMPRSS2/ERG promotes epithelial to mesenchymal transition through the ZEB1/ZEB2 axis in a prostate cancer model. PLoS One 6: e21650, 2011.

32. Cummins JM, He Y, Leary RJ, et al: The colorectal microRNAome. Proc Natl Acad Sci USA 103: 3687-3692, 2006.

33. Yanaihara N, Caplen N, Bowman E, et al: Unique microRNA molecular profiles in lung cancer diagnosis and prognosis. Cancer Cell 9: 189-198, 2006

34. Mott JL, Kobayashi S, Bronk SF and Gores GJ: mir-29 regulates Mcl-1 protein expression and apoptosis. Oncogene 26: 6133-6140, 2007.

35. Braconi C, Kogure T, Valeri N, et al: microRNA-29 can regulate expression of the long non-coding RNA gene MEG3 in hepatocellular cancer. Oncogene 30: 4750-4756, 2011.

36. Xiong Y, Fang JH, Yun JP, Yang J, Zhang Y, Jia WH and Zhuang SM: Effects of microRNA-29 on apoptosis, tumorigenicity and prognosis of hepatocellular carcinoma. Hepatology 51: 836-845, 2010 